\title{
Pitfalls in the determination of human acylated ghrelin plasma concentrations using a double antibody enzyme immunometric assay
}

Arjun Trivedi, Sandra Babic and Jean-Pierre Chanoine.

Department of Pediatrics, British Columbia Children's Hospital and Child and Family Research Institute, University of British Columbia, Vancouver, BC, Canada, V6H $3 \mathrm{~V} 4$

Correspondence: $\quad$ Arjun Trivedi

Child and Family Research Institute, A4-151 Bay 16

British Columbia's Children's Hospital, 4480 Oak Street,

Vancouver BC V6H 3V4 Canada

Phone: 1 (604) 875 2345, ext 6597

Fax: 1 (604) 8753231

E-mail: atrivedi@interchange.ubc.ca 


\begin{abstract}
:
Objectives: To investigate the effect of hemolysis and protease inhibitors on acylated ghrelin (AG) concentrations measured with a double antibody enzyme immunometric assay that uses an acetylcholinesterase (AChE)-Fab' conjugate.
\end{abstract}

Design and Methods: Samples were hemolysed or treated with PHMB (p-

hydroxymercuribenzoate), PMSF (phenylmethanesulfonylfluoride) or AEBSF (4-(2-Aminoethyl) benzenesulfonyl fluoride) to prevent AG degradation.

Results: Hemolysis decreased AG concentrations. PHMB or PMSF did not affect the assay. The standard curve was abolished by AEBSF but rescued by addition of a washing step prior to the AChE-Fab' conjugate.

Conclusions: Hemolysis and AEBSF may affect AG determination.

\title{
Key Words
}

Double antibody enzyme immunometric assay, Hemolysis, Protease Inhibitors, Acylated Ghrelin, Acetylcholinesterase

\begin{abstract}
Abbreviations
AG, acylated ghrelin; UAG, unacylated ghrelin; GOAT, ghrelin o-acyltransferase; EIA, enzyme immunometric assay; AChE, acetylcholinesterase; PHMB, p-hydroxymercuribenzoate; PMSF, phenylmethanesulfonylfluoride; AEBSF, 4-(2-Aminoethyl) benzenesulfonyl fluoride
\end{abstract}




\section{Introduction}

Ghrelin is a 28 amino acid peptide hormone that is thought to play an important physiological role in energy balance. It circulates as acylated (AG) and unacylated (UAG) ghrelin [1]. Acylation is necessary for its interaction with a G-protein coupled receptor to foster the release of growth hormone from the anterior pituitary gland. This is accomplished by addition of an octanoate group to the serine- 3 residue of ghrelin by the enzyme ghrelin-oacyltransferase (GOAT) [2]. In order to prevent the rapid deacylation and proteolysis of circulating AG [3] manufacturers of AG assays suggest that protease inhibitors be added to the blood sample immediately following collection and that the plasma be acidified.

Recently, a sensitive Enzyme Immunometric Assay (EIA) using a double-sandwich antibody technique has been developed for AG determination. The double-sandwich concept makes it possible to detect only full length molecules of ghrelin, contrasting with single antibody assays that also detect incomplete molecules. As a consequence, it provides lower AG concentrations and greater specificity [4]. It involves binding of the C-terminal part of the ghrelin molecule to a first, well-coated antibody followed by binding of an acetylcholinesterase (AChE) Fab' conjugate recognizing the N-terminal part of ghrelin. Thereafter, Ellman's reagent is added and reacts with the AChE tracer to form a yellow compound that is read at $414 \mathrm{~nm}$. We hypothesized that this assay could be affected by hemolysis or by protease inhibitors used to prevent AG deacylation. The objectives of the study were to assess the effect of various levels of hemolysis and of several commonly used protease inhibitors on human AG concentrations. 


\section{Materials and Methods}

Blood was collected from four healthy adult volunteers in prechilled EDTA tubes (Vacutainer, $1.8 \mathrm{mg}$ of K2 EDTA/ml blood, Becton Dickinson, Franklin Lakes, NJ, USA). In both experiments, tubes were centrifuged at $4^{\circ} \mathrm{C}(3500 \mathrm{rpm})$ and $100 \mu \mathrm{L}$ of $1 \mathrm{~N}$ hydrochloric acid $(\mathrm{HCl})$ was added to each $\mathrm{ml}$ of plasma prior to storage at $-20^{\circ} \mathrm{C}$. Acylated ghrelin was measured using a double sandwich antibody EIA (\# 10006306, Cayman Chemical Company, Ann Harbor, MI). All samples were run in duplicate.

\subsection{Hemolysis experiment}

Ten microL of a 100mM p-hydroxymercuribenzoate (PHMB) (\#55540, Sigma-Aldrich, St. Louis, MO) solution was added to each $\mathrm{mL}$ of blood prior to centrifugation to prevent $\mathrm{AG}$ degradation. Hemolysis was obtained mechanically by passing the blood through a 25 gauge needle an increasing number of times. The degree of hemolysis was assessed through determination of free hemoglobin $(\mathrm{g} / \mathrm{L})$ in the plasma sample and through visual comparison against a laboratory hemolysis chart. The assay was performed using a 1 in 5 dilution of the samples and a 24 hour incubation according to the recommendations of the manufacturer.

\subsection{Protease inhibitor experiment}

The following protease inhibitors were added to each $\mathrm{ml}$ of plasma: $10 \mathrm{microL}$ of $100 \mathrm{mM}$ PHMB in water (as recommended by Cayman) or $10 \mathrm{microL}$ of a $10 \mathrm{mg} / \mathrm{mL}$ phenylmethanesulfonylfluoride (PMSF) (\#36978, Thermo Scientific, Rockford, IL) methanol solution (as recommended by Millipore, Billerica, MA for assay GHRA-88HK) or 10microL of 100mg/mL 4-(2-Aminoethyl) benzenesulfonyl fluoride (AEBSF) (\#A8456, Sigma-Aldrich, St. Louis, MO) in water (as recommended by Millipore for assay EZGRT-89K). The assay was performed using a 1 in 5 dilution of the samples and a 6 hour incubation. One 96 well plate was used for each inhibitor. For each plate, we ran a normal standard curve and a standard curve where the protease inhibitor had been added to the standards at the same final concentration as for the samples. In addition, for the AEBSF plate, where the standard curve was markedly affected by the protease inhibitor, we ran an extra standard curve with one additional step of washing in an attempt to remove the protease inhibitor following the first incubation and to rescue the assay: following incubation with the first antibody for 3 hours, the wells were washed 
5 times with 300microL of the supplied wash buffer prior to addition of the $2^{\text {nd }}$ antibody (AchEFab'conjugate) for a total incubation time of 6 hours.

The study was approved by the University of British Columbia Clinical Ethics Review Board. Written informed consent was received from all participants.

\subsection{Statistics}

Results are presented as Mean (SD). Statistical analysis was performed after log transformation of the data. One-way ANOVA for repeated samples followed by posthoc t-test (comparing the plasma samples with various degrees of hemolysis to the non-hemolysed sample) was used to assess the effect of hemolysis on AG concentrations. Paired t-test was used to assess the differences in $\mathrm{AG}$ values measured according to the different standard curves. Linear regression (with the square of the Spearman coefficient $r$ ) was used to express the relationship between AG values in the different standard curves (SPSS version 17.0, Chicago, Ill., USA). A p $<0.05$ was taken as significant. 


\section{Results}

Plasma AG concentrations decreased progressively with increasing hemolysis (Table 1, P = $0.002)$.

Protease inhibitors had different effects on the standard curves. Compared to a standard curve performed according to the recommendations of the manufacturer, addition of PHMB and PMSF to the standards at the concentrations mentioned in section 2.2, did not affect the linear regression of the standard curve $\left(\mathrm{r}^{2} \geq 98\right.$ for all curves) (Fig 1A and 1B). In contrast, addition of AEBSF to the standards completely abolished the standard curve (Fig 1C). We assumed that this may be due to the inhibitory effect of AEBSF on AChE and attempted to rescue the curve by adding a washing step to remove the AEBSF prior to the addition of the second antibody (AChEFab' conjugate). This successfully rescued the standard curve (Fig 1C). We then compared the results obtained for PHMB- and AEBSF- treated plasma samples from 4 volunteers run according to the recommendations of the manufacturer and for AEBSF-treated plasma samples run using our 2 step rescue procedure. Samples were read against a control standard curve. Acylated ghrelin concentrations were 123 (145) pg/ml for PHMB-treated samples and undetectable for AEBSF-treated samples run according to the recommendations of the manufacturer. Mean (SD) concentrations were 118 (89) $\mathrm{pg} / \mathrm{ml}$ for AEBSF-treated samples run using our 2 step rescue process with an additional washing step. There was no significant difference between AG concentrations in PHMB-treated samples and in AEBSF-treated samples rescued with a two-step procedure and $(P=0.45)$. There was a trend towards a significant correlation between the results obtained with both methods $\left(\mathrm{r}^{2}=0.89, P=0.055\right)$. 


\section{Discussion}

Hemolysis is a common occurrence in clinical practice and did adversely affect the detectable AG concentrations in our study. This could potentially be due to an interference by the lysed red blood cells with the antigen-antibody interaction ( $1^{\text {st }}$ or $2^{\text {nd }}$ antibody) or to red blood cell peroxides that are known to inhibit $\mathrm{AChE}[5,6]$.

Several protease inhibitors have been recommended in order to prevent deacylation and degradation of AG. Conceptually, these inhibitors could affect the action of AChE and, as a consequence, the results of the double sandwich antibody assay used in the present study. The spectrum of action of protease inhibitors is not always clearly defined in the literature. PHMB, which is recommended by the manufacturer, is a cysteine protease inhibitor that does not interfere with AChE [7]. Accordingly, addition of PHMB to the standards did not affect the standard curve of the assay. PMSF was reported to inhibit not only proteases involved in AG degradation, but also AChE [8]. However, in our hands, addition of PMSF to the standards at a final concentration of $20 \mathrm{microg} / \mathrm{mL}$ or $5.6 \times 10^{-5} \mathrm{M}$ did not affect the standard curve, most likely because the PMSF concentration was too low to result in a complete AChE inhibition that has been shown to occur at $10^{-3} \mathrm{M}$ [8]. Finally, AEBSF did abolish the standard curve. AEBSF is a serine protease inhibitor known to inhibit AChE [9]. The addition of a washing step in order to remove the AEBSF present in the wells prior to addition of the AChE conjugate rescued the standard curve. This confirms our hypothesis that AEBSF prevented the reaction of the AChEFab' conjugate with the Ellman's reagent and the formation of a yellow compound. In summary, hemolysis affects the determination of $A G$, suggesting that the interpretation of $A G$ results from hemolysed samples may not be accurate. The use of AEBSF to prevent the degradation of $A G$ in plasma samples should be avoided when using an assay that includes an $\mathrm{AChE}$ conjugate as $2^{\text {nd }}$ antibody, although addition of an extra washing step may rescue the assay. These data are useful for researchers involved in ghrelin research. 


\section{Acknowledgements}

This study was supported by the Canadian Institutes for Health Research (CIHR).

\section{Disclosure}

The authors have no conflict of interest with any of the products mentioned in this manuscript 


\section{Figure legend}

Figure 1. Standard curves for human AG concentrations. Control curves were performed as per the recommendations of the manufacturer except for the duration of the incubation (6 hours). For PHMB (A), PMSF (B) and AEBSF (C) curves, protease inhibitors were added to the standards as described in section 2.2 and the assay otherwise performed as for the "control" curves.

The rescue AEBSF curve was performed by washing the first antibody with assay buffer after 3

hours of incubation, prior to adding the second antibody and incubating for another 3 hours (total incubation duration: 6 hours). 
Table 1: Mean (SD) concentrations and percent change in AG concentrations according to the degree of hemolysis assessed by visual inspection and by plasma hemoglobin determination

\begin{tabular}{|l|c|c|c|c|}
\hline Hemolysis & $\begin{array}{c}\text { Plasma Hb } \\
\text { concentration } \\
\text { (Visual inspection) } \\
(\mathbf{g} / \mathbf{L})\end{array}$ & $\begin{array}{c}\text { Plasma Hb } \\
\text { concentration } \\
\text { (Measured) } \\
\mathbf{( g / L )}\end{array}$ & $\begin{array}{c}\text { Mean (SD) } \\
\text { AG } \\
\text { concentrations } \\
(\mathbf{p m o l} / \mathbf{L}) *\end{array}$ & $\begin{array}{c}\text { Mean (SD) } \\
\text { decrease in } \\
\text { AG } \\
\text { concentrations } \\
\mathbf{( \% )}\end{array}$ \\
\hline No & 0.25 & 0.75 & $85.0(60.6)$ & Baseline \\
\hline Low & 1.81 & 2 & $79.3(58.6)$ & $7.8(6.0)$ \\
\hline Medium & 4.75 & 3.5 & $64.6(49.4) \#$ & $28.3(10.5)$ \\
\hline Severe & 5 & 4 & $61.8(52.7) \#$ & $34.7(14.8)$ \\
\hline
\end{tabular}

$\mathrm{N}=4$

$*: \mathrm{p}=0.02$, ANOVA for repeated samples after log transformation of the data.

\#: $\mathrm{p}<0.05$, compared to the "No hemolysis" group. 


\section{References}

[1] E. Tham, J. Liu, S. Innis, D. Thompson, B. Gaylinn, R. Bogarin, A. Haim, M. Thorner, J.P. Chanoine, Acylated ghrelin concentrations are markedly decreased during pregnancy in mothers with and without gestational diabetes: relationship with cholinesterase, AM. J Physiol. Endocrinol. Metab. 296 (2009) 1093-1100.

[2] T. Zhao, G. Liang, R. Lin Li, X. Xie, M. Sleeman, A.J. Murphy, D.M. Valenzuela, G.D. Yancopoulos, J.L. Goldstein, M.S. Brown, Ghrelin o-acyltransferase is essential for growth hormone mediated survival of calorie restricted mice, PNAS 107 (2010) 7467-7472.

[3] H. Ni, P. Walia, JP. Chanoine. Ontogeny of acylated ghrelin degradation in the rat, Peptides 31 (2010) 301-306.

[4] C. Prudom, J. Liu, J. Patrie, BD. Gaylinn, KE. Foster-Schubert, DE. Cummings, MO. Thorner, HM. Geysen, Comparison of competitive radioimmunoassay and two-site sandwich assays for the measurement and interpretation of plasma ghrelin levels, J Clin Endocrinol Metab 95 (2010) 2351-2358.

[5] B.W. O’Malley, C.E. Mengel, D. Meriwether, L.G. Zirkle, Inhibition of Erythrocyte Acetylcholinesterase by Peroxides, Biochemistry 5 (1966) 40-45.

[6] C. Giulivi, P. Hochstein, K.J.A Davies, Hydrogen peroxide production by red blood cells, Free Radical Biology \& Medicine 16 (1994) 123-129.

[7] J.S. Bond, P.E. Butler, Intracellular Proteases, Ann. Rev. Biochem. 56 (1987) 333-364.

[8] P. Turini, S. Kurooka, M. Steer, A.N. Corbascio, T.P. Singer, The action of phenylmethylsulfonyl fluoride on human acetylcholinesterase, chymotrypsin and trypsin, J. Pharmacology \& Experimental Therapeutics 167 (1969) 98-104.

[9] G.R. Mintz, An irreversible serine protease inhibitor, BioPharm 6 (1993) 34-38. 
Figure 1.
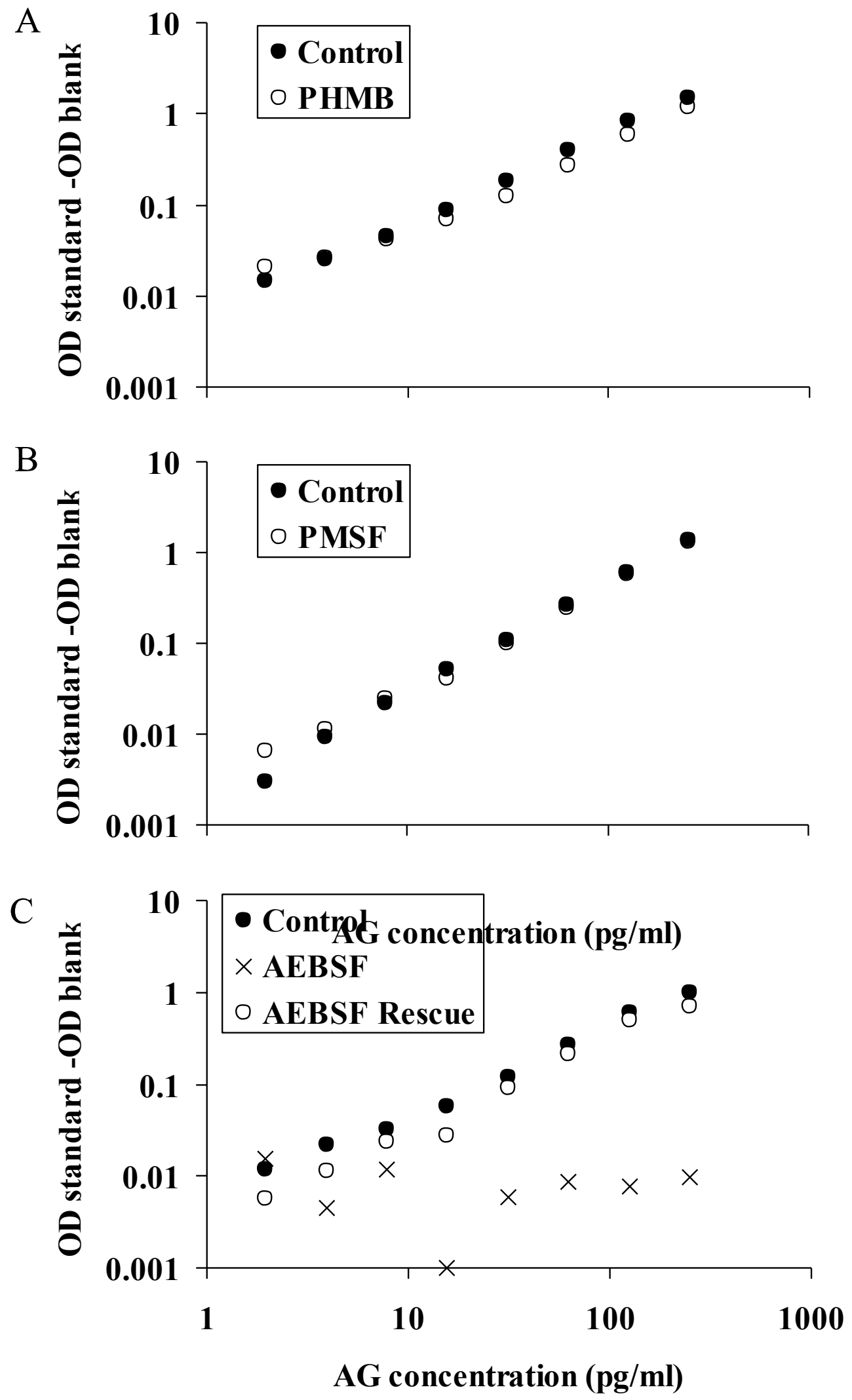